\title{
A vascular bed-specific pathway regulates cardiac expression of endothelial nitric oxide synthase
}

\author{
Pascale V. Guillot, ${ }^{1}$ Jason Guan, ${ }^{1}$ Lixin Liu, ${ }^{1}$ Jan A. Kuivenhoven, ${ }^{2}$ Robert D. Rosenberg, ${ }^{1,2}$ \\ William C. Sessa, ${ }^{3}$ and William C. Aird ${ }^{1}$ \\ ${ }^{1}$ The Department of Medicine, Divisions of Molecular Medicine and Hematology-Oncology, Beth Israel Deaconess Medical Center, \\ Boston, Massachusetts 02215, USA \\ ${ }^{2}$ Department of Biology, Massachusetts Institute of Technology, Cambridge, Massachusetts 02119, USA \\ ${ }^{3}$ Department of Pharmacology, Yale University School of Medicine, New Haven, Connecticut 06536, USA \\ Address correspondence to: William C. Aird, Division of Molecular Medicine, RW-663, Beth Israel Deaconess Medical School, \\ Boston, Massachusetts 02215, USA. Phone: (617) 667-1031; Fax: (617) 667-2913; E-mail: waird@bidmc.harvard.edu
}

Received for publication December 10, 1998, and accepted January 27, 1999.

\begin{abstract}
The endothelial nitric oxide synthase (eNOS) gene is induced by a variety of extracellular signals under both in vitro and in vivo conditions. To gain insight into the mechanisms underlying environmental regulation of eNos expression, transgenic mice were generated with the 1,600-bp 5' flanking region of the human eNos promoter coupled to the coding region of the LacZ gene. In multiple independent lines of mice, transgene expression was detected within the endothelium of the brain, heart, skeletal muscle, and aorta. $\beta$-galactosidase activity was consistently absent in the vascular beds of the liver, kidney, and spleen. In stable transfection assays of murine endothelial progenitor cells, the 1,600-bp promoter region was selectively induced by conditioned media from cardiac myocytes, skeletal myocytes, and brain astrocytes. Cardiac myocyte-mediated induction was partly abrogated by neutralizing anti-plateletderived growth factor (PDGF) antibodies. In addition, promoter activity was upregulated by PDGF-AB. Analysis of promoter deletions revealed that a PDGF response element lies between -744 and $-1,600$ relative to the start site of transcription, whereas a PDGF-independent cardiac myocyte response element is present within the first $166 \mathrm{bp}$ of the $5^{\prime}$ flanking region. Taken together, these results suggest that the eNos gene is regulated in the cardiac endothelium by both a PDGF-dependent and PDGF-independent microvascular bed-specific signaling pathway.
\end{abstract}

J. Clin. Invest. 103:799-805 (1999).

\section{Introduction}

The nitric oxide synthases (NOS) are a family of enzymes that mediate the biosynthesis of nitric oxide. There are three known isoforms: neuronal (nNOS, or NOS-I), inducible (iNOS, or NOS-II), and endothelial NOS (eNOS, or NOS-III) (1). eNOS is widely expressed within the endothelial lining of the vascular tree and is believed to play an important role in regulating vasomotor tone. Disruption of eNOS activity has been linked to the development of atherosclerosis and hypertension as well as an impairment in vascular remodeling and angiogenic response (2-6). The $5^{\prime}$ flanking region of the human eNOS gene has recently been cloned and sequenced (7). Under in vitro conditions, a 1,600-bp fragment was found to direct cell type-restricted expression in bovine aortic endothelial cells (BAECs). An Sp1 binding site at -103 relative to the start site of transcription was shown to be essential for basal activity of the promoter (7). However, endothelial cells grown in culture are uncoupled from critical environmental cues and may undergo significant phenotypic change. As a result, conclusions about transcriptional control mechanisms in vitro must be interpreted with caution. Indeed, the endogenous eNOS gene is now known to be induced by a variety of extracellular signals, including shear stress, hypoxia, estrogens, ischemia, exercise, vascular endothelial growth factor (VEGF), basic fibroblast growth factor (bFGF), and lysophosphatyl- choline (8-15). It follows that a full understanding of the transcriptional control mechanisms underlying eNOS expression will rely on model systems that preserve the endothelial cell's native environment.

To study the regulation of the eNOS gene in the context of an appropriate microenvironment, we generated transgenic mice with a construct containing the 1,600bp 5' flanking region of the eNOS promoter coupled to the coding sequence of $L a c Z$. In multiple independent lines of transgenic mice, reporter gene expression was indeed restricted to the endothelium. However, $\beta$-galactosidase activity was limited to a subpopulation of endothelial cells within the heart, skeletal muscle, brain, and aorta. Reporter gene activity was notably absent in other vascular beds that otherwise express the endogenous gene. In stable transfections assays, the eNOS promoter was selectively upregulated by conditioned media from cardiac myocytes, skeletal myocytes, and brain astrocytes. Induction of the eNOS promoter by cardiac myocytes was mediated by both platelet derived growth factor (PDGF)-dependent and PDGF-independent signaling pathways, whereas induction by skeletal myocytes and brain astrocytes was mediated by a PDGF-independent pathway. Taken together, the findings suggest that the eNOS gene is regulated by vascular bed-specific signaling pathways that begin in the extracellular milieu and end at distinct regions of the promoter. 


\section{Methods}

eNOS reporter gene constructs. The various human eNOS promoter constructs were derived from F1 LUC (7). To generate the transgenic construct $1600 \mathrm{eNOSlacZ}$, the KpnI-NotI promoter fragment $(-1,600$ to $+22 \mathrm{bp})$ was isolated from the F1 LUC vector and inserted into a plasmid-containing $L a c Z$ cDNA coupled to the SV40 poly(A) tail (gift from J. Rossant, Mount Sinai Hospital, Toronto, Canada). For stable transfections, the F1 LUC was digested with $S m a I$, releasing the -744 to $-1,600$ promoter fragment. The remaining vector was religated, resulting in $744 \mathrm{eNOS}$ LUC. The generation of the $166 \mathrm{eNOS}$ LUC (also referred to as F6 LUC) has been described previously (7).

Generation and analysis of transgenic mice. The $1600 \mathrm{eNOSlacZ}$ plasmid was digested with KpnI and NotI to release the Bluescript vector (Stratagene, La Jolla, California, USA), and the fragment consisting of the 1,600-bp eNOS promoter fragment coupled to $L a c Z$ was isolated on agarose gel. The DNA was resuspended in injection buffer and injected into fertilized mouse eggs as described previously (16). Transgenic founders were identified by Southern blot analysis using a $\left[{ }^{32} \mathrm{P}\right] \mathrm{dCTP}-$ labeled DNA probe containing LacZ coding sequence. The founders were bred to wild-type FVB mice to generate stable transgenic lines. Tissues were harvested from adult F1 offspring heterozygous for the transgene and processed for $L a c Z$ staining as described previously (16). For reverse transcriptase-PCR (RT-PCR), total RNA was harvested from various FVB mouse organs using a guanidinium thiocyanate phenol-chloroform single-step extraction (Stratagene). First-strand cDNA synthesis and PCR reactions were carried out as described previously, using primer sets specific for Escherichia coli LacZ and mouse eNOS (sense 5' ATCCACAGAACCTGTGGCCGTGGAACA; antisense 5' AGAATTCTCTGCACGGTTTGCAGGACG) (17)
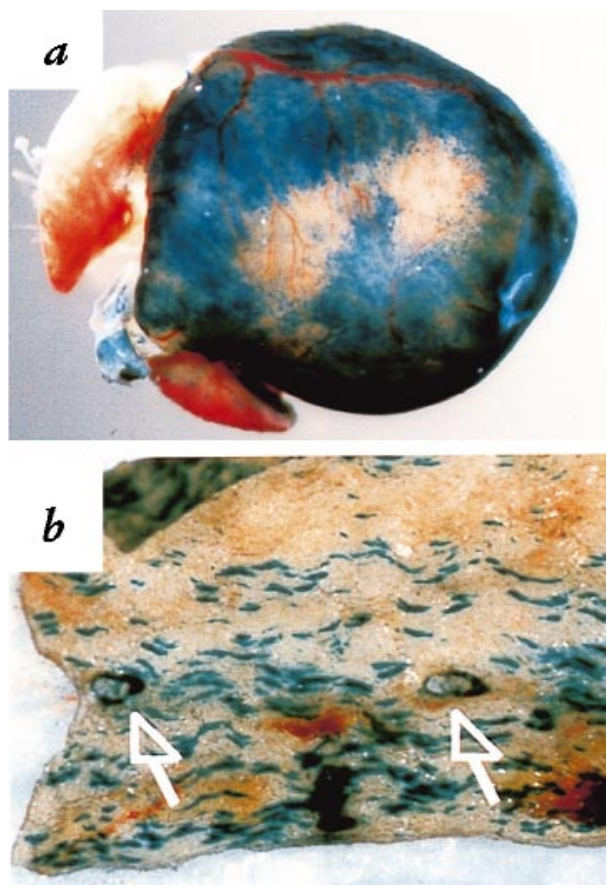

Figure 1

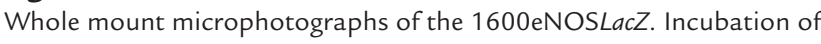
the organs with the X-Gal substrate reveals $L a c Z$ staining in the adult heart (a) and aorta (b). LacZ staining in the heart represents $\beta$-galactosidase activity within microvascular and macrovascular endothelial cells (see Fig. 2). The $X-G a l$ reaction product in the aorta is accentuated in endothelial cells surrounding the ostia of two intercostal tributaries ( $b$, arrows). eNOS, endothelial nitric oxide synthase.
Cell culture, DNA transfection, and treatments. Mouse embryonic endothelial progenitor (MEEP) cells were harvested and grown in DMEM (Life Technologies Inc., Gaithersburg, Maryland, USA) containing 20\% heat-inactivated FCS (Life Technologies Inc.) (18), as described previously. BAECs, HEK-293 cells (ATCC CRL-1573), H9c2 rat cardiac myocytes (ATCC CRL-1446), L8 rat skeletal myocytes (ATCC CRL-1769), DI TNC1 rat astrocytes (ATCC CRL-2005), and HepG2 cells (ATCC CRL-10741) were maintained in DMEM containing 10\% heat-inactivated FCS. All cells were cultured at $37^{\circ} \mathrm{C}$ and $5 \% \mathrm{CO}_{2}$. For stable transfections, $10^{7}$ MEEP cells were electroporated with a total of $30 \mu \mathrm{g}$ of Sall-linearized promoter construct and 1:10 molar ratio of DraI-linearized PGK-neo (Gene Pulser II; 350V, 500 F; Bio-Rad Laboratories Inc., Hercules, California, USA). The cells were grown for 9 days on 10-cm gelatin-coated plates in G418-containing medium ( $400 \mu \mathrm{g} / \mathrm{ml}$; Sigma Chemical Co., St. Louis, Missouri, USA). G418resistant clones (>200 per plate) were then trypsinized, pooled, and grown to confluence (P1). Luciferase assays were performed on cells below third passage. Cells were seeded onto 12-well plates, grown to confluence, and then incubated in DMEM $/ 0.2 \% \mathrm{FCS}$ for $24 \mathrm{~h}$. The serum-starved cells were then incubated for 4-24 h with DMEM/0.2\% FCS containing either $10 \mathrm{ng} / \mathrm{ml}$ PDGF-AA (PeproTech Inc., Rocky Hill, New Jersey, USA), PDGF-AB (R\&D Systems, Minneapolis, Minnesota, USA), PDGF-BB (PeproTech Inc.), 10 ng/ml VEGF (R\&D Systems), $10 \mathrm{ng} / \mathrm{ml} \mathrm{bFGF} \mathrm{(Sigma} \mathrm{Chemical}$ Co.), or $10 \mathrm{ng} / \mathrm{ml}$ epidermal growth factor (EGF) (R\&D Systems). Alternatively, the cells were incubated for 4-24h with conditioned medium from cardiac myocytes, skeletal myocytes, brain astrocytes, or HEK or HepG2 cells (70\% confluence and medium 2-3 days old) in the absence or presence of neutralizing antibodies to PDGF, VEGF, and bFGF (R\&D Systems). Cells were processed in triplicate for luciferase assay according to the manufacturer's instructions (Promega Corp., Madison, Wisconsin, USA), and light activity was measured in a luminometer (Lumat LB 9507; EG\&G Berthold, Bad Wildbad, Germany). In addition, cell number was determined in triplicate. In multiple independent experiments, there was no statistically significant difference in cell number under the conditions just described.

Northern blot and Western blot analyses. BAECs were serum starved in DMEM/0.5\% FCS for $24 \mathrm{~h}$, incubated for $2-8 \mathrm{~h}$ in the absence or presence of PDGF-AB, VEGF, bFGF, or EGF, and then harvested for total RNA using a guanidinium thiocyanate phenol-chloroform single-step extraction (Stratagene). The isolated RNA (5 ug) was loaded on a $0.7 \%$ formaldehyde-containing agarose gel. The RNA was transferred to nylon membrane, covalently cross-linked with ultraviolet radiation, prehybridized for $6 \mathrm{~h}$, and then hybridized for $18 \mathrm{~h}$ at $42^{\circ} \mathrm{C}$ with a $\left.{ }^{32} \mathrm{P}\right] \mathrm{dCTP}-$ labeled cDNA probe containing full-length bovine eNOS sequence. Total protein was obtained from FVB mouse tissues, quantified, subjected to SDS-PAGE, and transferred to nitrocellulose membrane. The membrane was blocked overnight in 5\% skim milk and incubated with $1 \mathrm{ug} / \mathrm{ml}$ polyclonal antieNOS antibody (Transduction Laboratories, Lexington, Kentucky, USA) for $12 \mathrm{~h}$ at room temperature. The membrane was subsequently incubated with anti-mouse IgG antibody conjugated to horseradish peroxidase. Peroxidase activity was viewed using an enhanced chemiluminescence substrate system.

\section{Results}

Transgenic mice. We generated transgenic mice with a $1,600-$ bp eNOS promoter fragment coupled to the $L a c Z$ reporter gene (1600eNOSlac $Z$ ). In four independent lines of mice, the $\mathrm{X}-\mathrm{Gal}$ reaction product was detected in the endothelial lining of blood vessels within the heart, the brain, and the skeletal muscle, as well as the aorta (Figs. 1 and 2). In crosssections of coronary arteries, $\beta$-galactosidase activity was present in only a subpopulation of endothelial cells (Fig. 2, 

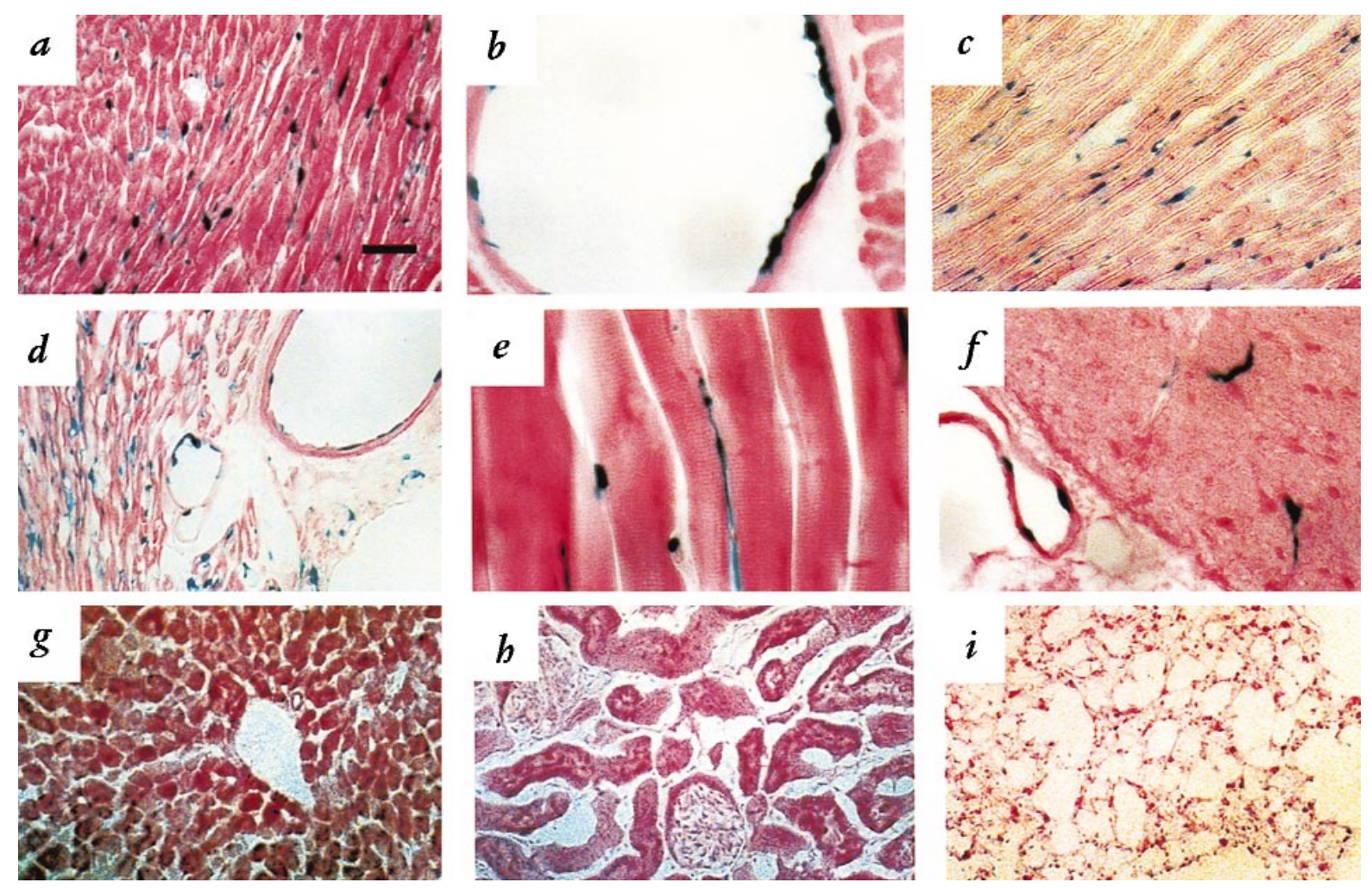

Figure 2

The $1600 \mathrm{eNOSLacZ}$ transgene is expressed in blood vessels of the heart, brain, and skeletal muscle. LacZ staining of 9- $\mu \mathrm{m}$ tissue sections from 1600 eNOS LacZ line 338-8 reveals reporter gene activity in the endothelial lining of cardiac microvessels $(\boldsymbol{a}, \boldsymbol{d})$, coronary arteries $(\boldsymbol{b})$, skeletal muscle $(\boldsymbol{e})$, and brain $(\boldsymbol{f})$. There is no detectable $\beta$-galactosidase activity in cardiac myocytes $(a)$, liver $(\boldsymbol{g})$, kidney $(\boldsymbol{h})$, and lung (i). Similar Lac $Z$ staining pattern in cardiac microvessels from transgenic line 338-41 can be seen in $\boldsymbol{c}$. Scale bar: $60 \mu \mathrm{m}$.

$b$ and $d$ ) The coronary veins and endocardium did not contain the X-Gal reaction product. Moreover, the X-Gal reaction product was not detected in cardiac myocytes. In one of the four lines, $L a c Z$ activity was also detected in the larger blood vessels of the lung (data not shown). In another of the four lines, the transgene was expressed ectopically in a subpopulation of neurons within the cortex of the brain. These latter findings suggest that the eNOSlac $Z$ transgene is susceptible to the influence of surrounding heterochromatin structure. $\beta$-galactosidase activity was consistently absent in the vascular beds of the kidney, spleen, and liver (Fig. 2, g-i). In RT-PCR analyses, Lac $Z$ transcripts were detected within the brain, skeletal muscle, and heart (Fig. 3). In contrast, endogenous eNOS mRNA and protein were present in all organs examined (Fig. 3).

Taken together, these findings indicate that the eNOS promoter fragment directs expression to a limited population of endothelial cells in the heart, skeletal muscle, brain, and aorta. The results suggest that the 1,600-bp promoter contains transcriptional control elements that are responsive to vascular bed-specific signaling pathways and that more proximal or distal DNA elements outside this region are responsible for expression in other vascular beds such as the kidney, spleen, and liver.

The 1,600-bp eNOS promoter is responsive to cardiomyocytederived signals in vitro. In an effort to elucidate the signals involved in regulating vascular bed-specific expression of eNOS, an in vitro tissue culture system was established to study transcriptional control in the context of the endothelial cell's microenvironment. In these studies, eNOS promoter constructs were stably transfected into mouse embryonic endothelial precursor cells derived from embryos at E7.5 (18). In multiple independent experiments involving more than six separate pools of stable transfectants, the 1,600-bp promoter was shown to be induced at four hours by conditioned media from cardiac myocytes, skeletal myocytes, and brain astrocytes (2.5- to 10-fold, mean 5.1-fold; 3.7- to 5.8-fold, mean 4.5fold; 3- to 4-fold, mean 3.7-fold, respectively) (Fig. 4a). In contrast, there was no change in expression levels in the presence of conditioned medium from either HEK or HepG2 cells, derived from kidney and liver parenchyma, respectively (Fig. 4a). These results are consistent with the expression pattern of the transgene in vivo and suggest that the eNOS promoter contains information for selective induction in the heart, skeletal muscle, and brain.

In a previous study (17), a fragment of the human von Willebrand factor (vWF) promoter was shown to direct environmentally regulated expression to the microvascular bed of the heart. We were interested in determining whether the eNOS gene was similarly regulated by a cardiac-specific signaling pathway. Under in vitro conditions, eNOS expression is induced by bFGF and $\operatorname{VEGF}(19,20)$. However, the incubation of cardiac myocyte-conditioned medium with neutralizing antibodies to VEGF and bFGF failed to reduce levels of induction (not shown). Moreover, the addition of VEGF and/or bFGF to unconditioned culture medium at concentrations between 10 and 100 


\section{Figure 3}

$\beta$-galactosidase activity correlates with LacZ mRNA levels. RT-PCR analysis of LacZ, and RT-PCR and Western blot analyses of endogenous eNOS in 1600 eNOS LacZ mouse tissues, reveal the presence of detectable $\beta$-galactosidase transcripts within the heart, brain, skeletal (sk) muscle, and to a lesser extent the lung, whereas eNOS transcripts and protein are present in all organs. For RT-PCR, each lane represents an RT-PCR analysis from identical DNA template. $\beta$-actin is included as a control for RNA levels. For Western blot analysis, the $140-\mathrm{kDa}$ control eNOS is a positive control derived from human aortic endothelial cell lysates. $R T-P C R$, reverse transcriptase-PCR.
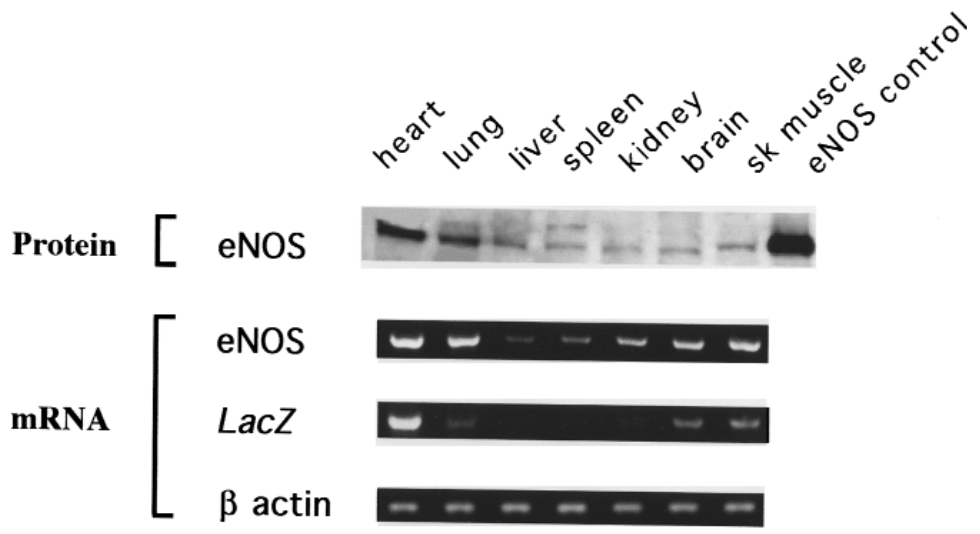

$\mathrm{ng} / \mathrm{ml}$ did not increase luciferase activity above baseline values (Fig. 4b). These data argue against a role of either VEGF or bFGF in mediating induction of the eNOS promoter by cardiac myocytes. Other studies have shown that PDGF signaling pathways play a critical role in the development and trabeculation of the murine heart (21-25). More recently, the PDGF-AB heterodimer has been shown to induce a network of genes within the microvascular bed of the heart, including the vWF and protein tyrosine kinase receptor Flk-1 (26). To test whether PDGF might be responsible for the cardiac myocyte-dependent induction of eNOS expression, the stable transfection pools were incubated with cardiac myocyte-conditioned medium containing neutralizing anti-PDGF antibodies. As shown in Fig. 4b, the cardiac myocyte-mediated induction of luciferase was significantly blunted in the presence of these antibodies (from mean 5.1-fold to 3-fold). In other experiments, the addition of PDGF-AB to unconditioned medium resulted in a significant increase in luciferase activity (2.4- to 5-fold, mean 3.7-fold) (Fig. 4b). PDGF-AA or PDGF-BB also induced promoter activity, but to a lesser extent than PDGF-AB (data not shown). Taken together, these results suggest that the eNOS promoter responds to cardiac myocyte-conditioned medium, in part, through a PDGF-dependent signaling pathway. Importantly, the addition of PDGF antibodies, at concentrations that effectively suppressed the promoter response to PDGF 10 $\mathrm{mg} / \mathrm{ml}$, did not completely abrogate the cardiac myocytemediated induction of eNOS expression (Fig. 4b). The residual threefold induction of eNOS in the presence of anti-PDGF antibodies suggests that promoter activity in the heart is also regulated by a PDGF-independent signaling pathway.

To test whether PDGF activation of the eNOS promoter was a unique property of cardiac myocyte-conditioned medium, the stable transfection pools were incubated with conditioned media from skeletal myocytes or brain astrocytes in the presence or absence of neutralizing anti-PDGF antibodies. As shown in Fig. $4 b$, induction of the eNOS promoter was not attenuated by the addition of the antibodies. These results suggest that induction of eNOS promoter activity by the PDGF signaling pathway is specific to the heart.

To identify the promoter regions that direct cardiac myocyte-inducible expression of eNOS, two additional promoter luciferase constructs (744eNOS LUC and 166 eNOS LUC) were transfected into MEEP cells. As shown in Fig. 4c, 744eNOS LUC did not respond to PDGF-AB but was induced by cardiac myocyte-conditioned medium (1.3- to 2.2-fold, mean 1.7-fold). Similarly, the shorter 166eNOS LUC construct did not respond to PDGF but was upregulated in the presence of cardiac myocyte-conditioned medium (2- to 4.2-fold, mean 2.6-fold) (Fig. 4d). By contrast, the promoterless PGL2 vector failed to respond to PDGF-supplemented or cardiac myocyte-conditioned medium in stable transfection assays (data not shown). Taken together, these results indicate that the PDGF response element lies between -744 and $-1,600$ relative to the start site of transcription and that the response element(s) for the PDGFindependent signaling pathway is contained within the first $166 \mathrm{bp}$ of the $5^{\prime}$ flanking region.

eNOS is a PDGF-responsive gene. The results just described suggest that the 5' flanking region of the eNOS gene contains a PDGF-responsive element. To determine whether the endogenous eNOS gene is regulated by a PDGF signaling pathway, we performed Northern blot analyses of endothelial cells grown in the absence and presence of PDGF-AB. Under these conditions, eNOS transcripts were not detectable in MEEP cells (data not shown). However, PDGF-AB at a concentration of $10 \mathrm{ng} / \mathrm{ml}$ did result in a 2.4-fold induction of eNOS mRNA levels in BAEC at four hours (Fig. 5, $a$ and $b$ ). The induction was comparable to that of VEGF and somewhat less than the FGF- $\beta$ response (Fig. 5b). The addition of EGF had no effect on expression levels (Fig. 5b). A time course showed induction levels to be maximal at four hours (Fig. $5 a$ ). These results suggest that the endogenous eNOS gene is responsive to PDGF-AB and support the notion that the promoter is regulated by a PDGF-dependent pathway in vivo.

\section{Discussion}

The endothelium plays a pivotal role in mediating hemostasis, vascular wall tone, and trafficking of cells and nutrients between blood and underlying tissue. At the same time, the cardiovascular system is characterized by a remarkable array of organ- and region-specific endothelial cell properties that collectively give rise to functional specialization of the endothelium (27-29). For example, the postcapillary high-venuole endothelial cells in lymphoid organs support the binding and migration of lymphocytes via the specific interaction of adhesion molecules with lymphocyte homing receptors (30, $31)$, whereas the endothelial cells that line the small 
a

$b$
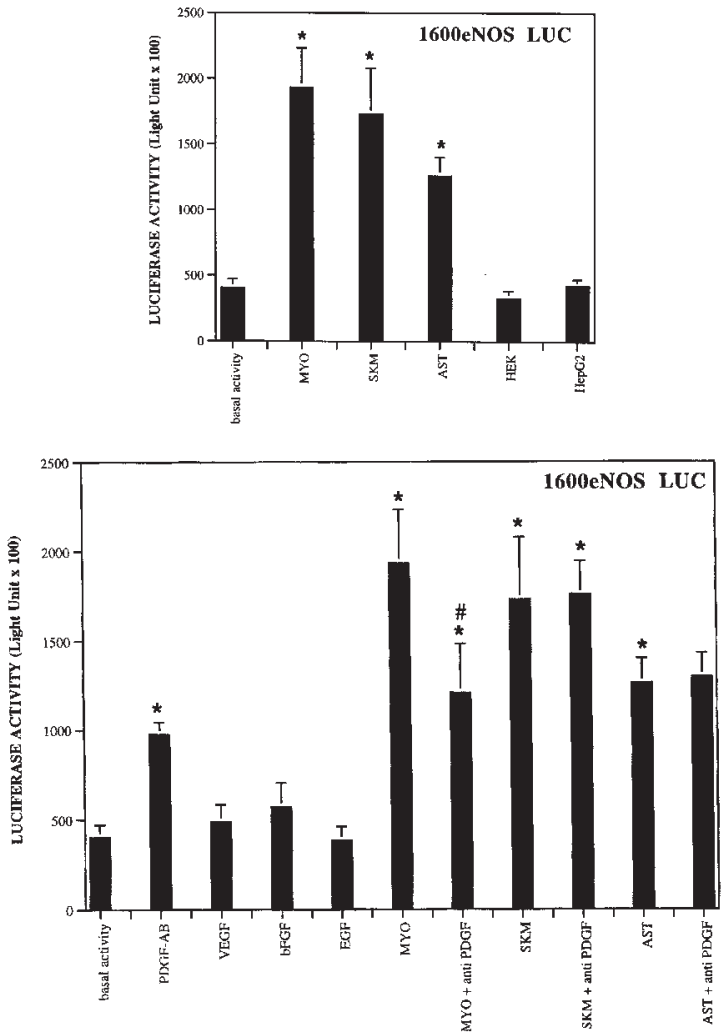

c

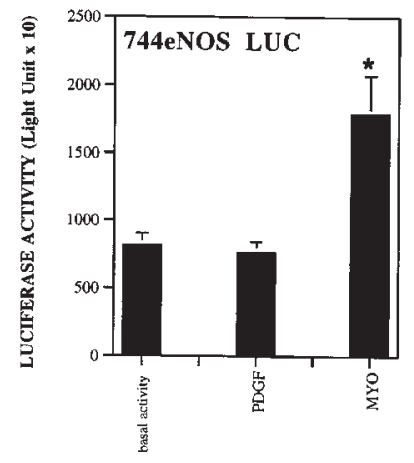

d

Figure 4

Expression of the $1600 \mathrm{eNOS}$ promoter is induced by myocyte-derived signaling pathways in vitro. MEEP cells were stably transfected with $1600 \mathrm{eNOS}$ LUC, 744eNOS LUC, and 166 eNOS LUC as described in Methods. Cells were starved in $0.2 \%$ FCS for $24 \mathrm{~h}$ and then incubated for $4 \mathrm{~h}$ in conditioned medium from HEK, HepG2, cardiac myocytes (MYO), skeletal myoctes (SKM), or brain astrocytes (AST) in the absence (a) or presence (b) of antiPDGF antibodies, or in media supplemented with PDGF, VEGF, bFGF, and EGF at concentrations of $10 \mathrm{ng} / \mathrm{ml}(b)$. The results show the mean and SD of luciferase light units obtained in triplicate from one representative experiment. The 1600eNOS LUC construct was induced 4.8-fold by cardiac myocyte-conditioned medium, 4.3-fold by skeletal muscle-conditioned medium, 3-fold by astrocyte-conditioned medium, 2.4-fold by PDGF-AB, and 3 -fold by cardiac myocyte-conditioned medium containing anti-PDGF antibodies ( $b$ ). The 744eNOS LUC construct was induced 2.2-fold, and the $166 \mathrm{eNOS}$ LUC construct 2.7 -fold, by myocyte-conditioned medium ( $\boldsymbol{c}$ and $\boldsymbol{d}$, respectively). ${ }^{*} P<0.05$ vs. basal activity; ${ }^{\#} P<0.05$ vs. cardiac myocyte-conditioned medium. $b F G F$, basic fibroblast growth factor; $E G F$, epidermal growth factor; $M E E P$, mouse embryonic endothelial progenitor; PDGF, platelet-derived growth factor; VEGF, vascular endothelial growth factor.

blood vessels of the brain possess a unique expression pattern of cell surface receptors, transporters, and intracellular enzymes that serve to regulate tightly the exchange of solutes between blood and brain parenchyma $(32,33)$. Distinct endothelial cell phenotypes have also been described in the liver, kidney, lung, and bone marrow (34-37). As an important corollary to phenotypic diversity, subpopulations of endothelial cells may respond in unique ways to pathophysiological stimuli. Indeed, it is quite likely that cell subtype-specific alterations in cell function contribute to the pathophysiology of focal vasculopathic disease states.

The central role that endothelial cell heterogeneity plays in establishing both normal and pathological vascular patterns underscores the importance of understanding how phenotypic diversity is generated. Previous investigations have shown that differential gene regulation in the endothelium is largely governed by environmental stimuli. For example, in vitro culture and in vivo transplant studies have documented the ability of astrocytes to induce the blood-brain barrier phenotype (38-42). Aortic endothelial cells may be induced to express Lu-ECAM-1 in the presence of lung-derived extracellular matrix and to acquire fenestrated gap junctions on kidney-derived matrix $(43,44)$. The critical role of extracellular signals in controlling vascular bed-specific gene expression was demonstrated in a recent transgenic study of the vWF promoter (17). In that study, a small region of the human vWF promoter directed expression to a subset of microvascular endothelial cells within the murine heart, skeletal muscle, and brain. More importantly, the restricted expression pattern was shown to be governed by signals residing in the local microenvironment (17).

In the current report, a 1,600-bp fragment of the eNOS promoter was found to direct expression to a similar subset of microvascular endothelial cells. The overlapping expression pattern of the eNOS and vWF transgenes raises the possibility that the two promoters are coregulated in the endothelium of the brain, heart, and skeletal muscle. In contrast to the vWF transgene, the eNOS promoter contained information for expression in the endothelial lining of the coronary arteries and aorta. Interestingly, the pattern of expression in these larger blood vessels was not uniform. For example, in the aorta, LacZ-positive cells 
$a$

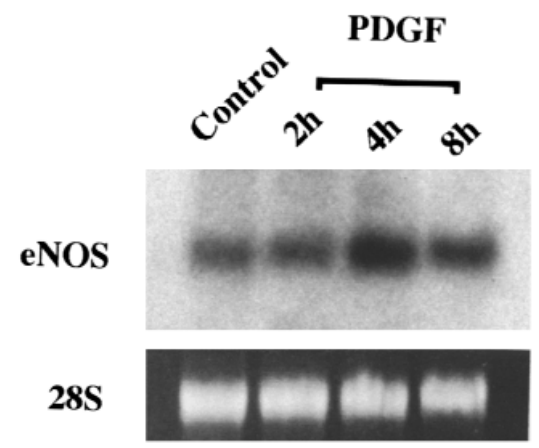

$\boldsymbol{b}$

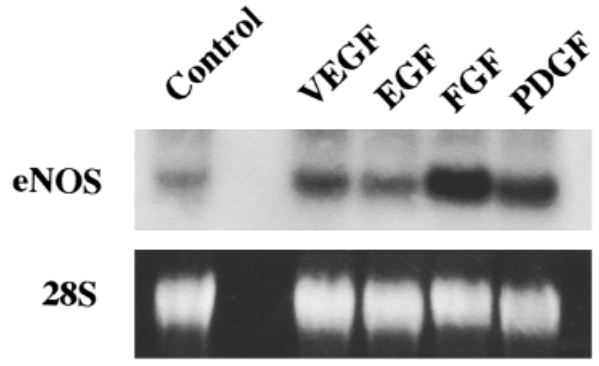

Figure 5

eNOS RNA levels are increased by PDGF. BAECs were starved in 0.5\% FCS for $24 \mathrm{~h}$ and then incubated with either VEGF, EGF, bFGF, or PDGF-AB at $10 \mathrm{ng} / \mathrm{ml}$ for $4 \mathrm{~h}$. Total RNA $(5 \mu \mathrm{g})$ was subjected to agarose electrophoresis. (a) Autoradiogram with an eNOS CDNA probe. (b) The ethidium-stained $28 \mathrm{~S}$ ribosomal band. BAECs, bovine aortic endothelial cells.

were aligned in clusters along the longitudinal axis of blood flow and were accentuated around the ostia of aortic tributaries. In cross-sections of the epicardial coronary arteries, the X-Gal reaction product rarely covered the full circumference of the blood vessel. As much as these findings may reflect variegate expression of the transgene or arise from artifacts from tissue processing, they are also consistent with microheterogeneity of the surrounding environment. Indeed, it is tempting to speculate that differential activity of the transgene within the aorta and coronary arteries is governed by local variation in fluid shear stress. Such a finding would complement previous demonstrations of flow-regulated expression of the eNOS gene under in vitro conditions (14-15).

Only one of the four expressing lines of mice contained $\beta$-galactosidase activity within the vascular bed of the lung. This finding indicates that expression of the eNOSlacZ transgene may be influenced by the site of genomic integration. It also raises the possibility that the 1,600-bp promoter contains a lung vascular bed-specific transcriptional control element that is active in the context of a permissive chromatin environment. The consistent absence of Lac $Z$ expression in the vascular beds of the kidney, spleen, and liver in each of the four lines contrasts sharply with the presence of endogenous eNOS mRNA in these organs. Discordant expression of the transgene and endogenous gene implies that eNOS is regulated by different mechanisms from one vascular bed to another. Although DNA sequences within the 1,600-bp promoter contain information for expression in the heart, skeletal muscle, brain, and perhaps the lung, more distal or prox- imal transcriptional control elements may be necessary for expression in the LacZ-negative vascular beds. Finally, the lack of detectable transgene expression in other eNOS expressing lineages such as cardiomyocytes and bronchial epithelial cells suggests that additional promoter elements are responsible for transcriptional activation in these cell types as well (45-47).

To screen for environmentally responsive promoter elements, an in vitro system was developed to monitor endothelial cell gene expression in the presence or absence of extracellular stimuli. Toward this end, eNOS promoter constructs were stably transfected into MEEP cells derived from murine embryos at E7.5 (18). These cells were used for several reasons. First, stable transfection protocols yield high numbers of clones that can be assayed at early passage. Second, the relatively undifferentiated state of the cells may render them more responsive to environmental manipulation in tissue culture. Finally, previous studies have shown that these cells incorporate into blood vessels of the developing heart, suggesting that they have the capacity to differentiate within a cardiac microenvironment (18). In Northern blot analyses, eNOS mRNA was not detectable in MEEP cells. The absence of endogenous transcripts suggests either that additional extracellular signals are lacking in the culture media, that negatively acting transcription factors are interacting with DNA elements outside the 1,600-bp promoter, or that epigenetic phenomena such as DNA methylation and heterochromatin configuration are operating to suppress expression. In this study, the MEEP cells were used as a tool to analyze eNOS promoter fragments outside the context of the fulllength gene and to track the vascular bed-specific pathways under in vitro conditions. In these experiments, activity of the 1,600-bp eNOS promoter was specifically induced by conditioned media from cardiac myocytes, skeletal myocytes, and brain astrocytes. The effect of cardiac myocytes was mediated by two distinct signaling pathways. The first involved the interaction of a PDGFdependent pathway with DNA sequences between -744 and $-1,600$ relative to the start site of transcription. The second involved a PDGF-, VEGF- and bFGF-independent pathway operating through the proximal $166 \mathrm{bp}$ of the promoter. As far as we know, this is the first study to demonstrate that the eNOS gene is responsive to PDGF. The biologic plausibility of PDGF-mediated gene expression in the microcirculation of the heart is supported by three lines of evidence. First, previous investigations have documented a critical role for PDGF-A, PDGF-B, and the PDGF-R $\alpha$ in the development of the heart and cardiac vasculature (21-25). Second, cardiac pathophysiology has been linked to abnormalities in PDGF signaling (48-50). Finally, PDGF-AB heterodimer has been shown to regulate a network of hemostatic and angiogenic genes within the heart, including vWF and Flk-1 (26). The results of the present study suggest that the eNOS gene is similarly regulated by a cardiac microvascular bed-specific circuit. It will be necessary to confirm these findings in appropriate in vivo model systems. It will be important not only to determine the relative roles of the PDGF-dependent and PDGF-independent pathways in mediating physiological expression of eNOS but also to characterize the upstream and downstream components of the cardiac-specific cir- 
cuits. Taken together, these various approaches should provide important insight into the physiology and pathophysiology of the cardiac microcirculation.

In summary, the results of this study suggest that the eNOS gene is regulated by a series of vascular bed-specific signaling pathways that begin in the extracellular milieu and end at distinct regions of the promoter. Overall temporal and spatial expression of eNOS within the vascular tree is determined by the net activity of environmentally responsive transcriptional activation modules. We have provided evidence that eNOS expression in the microvascular bed of the heart is mediated by cardiomyocyte-derived signals. We predict that expression of eNOS in other microenvironments is governed by the interaction of additional signaling pathways with other response modules. Using the complementary in vitro and in vivo approaches described in this study, we believe that we now have the means to map these response elements and to characterize the signaling pathways involved.

\section{Acknowledgments}

We thank Michael Simons and Linda Fritze for their careful review of the manuscript. We thank Tom Michel for providing us with the bovine eNOS cDNA probe. W.C. Sessa is supported by National Institutes of Health (NIH) grants HL-51948 and HL-57665. W.C. Aird is supported by NIH grant HL-59316.

1. Sessa, W.C. 1994. The nitric oxide synthase family of proteins. J. Vasc. Res 31:131-143.

2. Huang, P.L., et al. 1996. Hypertension in mice lacking the gene for endothelial nitric oxide synthase. Nature. 377:239-242.

3. Shesely E.G., et al. Elevated blood pressures in mice lacking endothelial nitric oxide synthase. 1996. Proc. Natl. Acad. Sci. USA. 93:13176-13181.

4. Wever, R.M., Luscher, T.F., Cosentino, F., and Rabelink, T.J. 1998. Atherosclerosis and the two faces of endothelial nitric oxide synthase. Circulation. 97:108-112.

5. Rudic, R.D., et al. 1998. Direct evidence for the importance of endotheliumderived nitric oxide in vascular remodeling. J. Clin. Invest. 101:731-736.

6. Murohara, T., et al. 1998. Nitric oxide synthase modulates J. Clin. Invest. 11:2567-2578.

7. Zhang, R., Min, W., and Sessa, W.C. 1995. Functional analysis of the human endothelial nitric oxide synthase promoter. Sp1 and GATA factors are necessary for basal transcription in endothelial cells. J. Biol. Chem. 270:15320-15326.

8. Sessa, W.C., et al. 1994. Chronic exercise in dogs increases coronary vascular nitric oxide production and endothelial cell nitric oxide synthase gene expression. Circ. Res. 74:349-353.

9. Zembowicz, A., Tang, J.L., and Wu, K.K. 1995. Transcriptional induction of endothelial nitric oxide synthase type III by lysophosphatidylcholine.J. Biol. Chem. 270:17006-17010.

10. Ranjan, V., Xiao, Z., and Diamond S.L. 1995. Constitutive NOS expression in cultured endothelial cells is elevated by fluid shear stress. Am. J. Physiol. 269:H550-H555.

11. Uematsu, M., et al. 1995. Regulation of endothelial cell nitric oxide synthase mRNA expression by shear stress. Am. J. Physiol. 269:C1371-C1378.

12. Arnet, U.A., et al. 1996. Regulation of endothelial nitric-oxide synthase during hypoxia. J. Biol. Chem. 271:15069-15073.

13. Nadaud, S., et al. 1996. Sustained increase in aortic endothelial nitric oxide synthase expression in vivo in a model of chronic high blood flow. Circ. Res. 79:857-863.

14. MacRitchie, A.N., et al. 1997. Estrogen upregulates endothelial nitric oxide synthase gene expression in fetal pulmonary artery endothelium. Circ. Res. 81:355-362.

15. Gess, B., Schricker, K., Pfeifer, M., and Kurtz, A. 1997. Acute hypoxia upregulates NOS gene expression in rats. Am. J. Physiol. 273:R905-R910.

16. Aird, W.C., et al. 1995. Human von Willebrand factor gene sequences target expression to a subpopulation of endothelial cells in transgenic mice. Proc Natl. Acad. Sci. USA. 92:4567-4571.

17. Aird, W.C., et al. 1997. Vascular bed-specific expression of an endothelial cell gene is programmed by the tissue microenvironment. J. Cell Biol. 138:1117-1124.

18. Hatzopoulos, A.K., et al. 1998. Isolation and characterization of endothelial progenitor cells from mouse embryos. Development. 125:1457-1468.
19. Kostyk, S.K., et al. 1995. Basic fibroblast growth factor increases nitric oxide synthase production in bovine endothelial cells. Am. J. Physiol. 269:H1583-H1589.

20. Hood, J.D., Meininger, C.J., Ziche, M., and Granger, H.J. 1998. VEGF upregulates ecNOS message, protein, and $\mathrm{NO}$ production in human endothelial cells. Am. J. Physiol. 274:H1054-H1058.

21. Stephenson, D.A., et al. 1991. Platelet-derived growth factor receptor alphasubunit gene (Pdgfra) is deleted in the mouse patch $(\mathrm{Ph})$ mutation. Proc. Natl. Acad. Sci. USA. 88:6-10.

22. Schatteman, G.C., et al. 1992. Regulation and role of PDGF receptor alphasubunit expression during embryogenesis. Development. 115:123-131.

23. Leveen, P., et al. 1994. Mice deficient for PDGF B show renal, cardiovascular, and hematological abnormalities. Genes Dev. 8:1857-1887.

24. Schattemann, G.C., Loushin, C., Li, T., and Hart, C.E. 1996. PDGF-A is required for normal murine cardiovascular development. Dev. Biol. 176:133-142.

25. Lindahl, P., Johansson, B.R., Leveen, P., and Betsholtz, C. 1997. Pericyte loss and microaneurysm formation in PDGF-B-deficient mice. Science. 277:242-245.

26. Edelberg, J.M., et al. 1998. PDGF mediates cardiac microvascular communication. J. Clin. Invest. 102:837-843.

27. Gerritsen, M.E. 1987. Functional heterogeneity of vascular endothelial cells. Biochem. Pharmacol. 36:2701-2711.

28. Page, C., Rose, M., Yacoub, M., and Pigott, R. 1992. Antigenic heterogeneity of vascular endothelium. Am. J. Pathol. 141:673-683.

29. Cines, D.B., et al. 1998. Endothelial cells in physiology and in the pathophysiology of vascular disorders. Blood. 91:3527-3561.

30. Streeter, P.R., et al. 1988. A tissue-specific endothelial cell molecule involved in lymphocyte homing. Nature. 331:41-46.

31. Berg, E.L., et al. 1989. Homing receptors and vascular addressins: cell adhesion molecules that direct lymphocyte traffic. Immunol. Rev. 108:5-18.

32. Bradbury, M.W. 1993. The blood-brain barrier. Exp. Physiol. 78:453-472.

33. Schlosshauer, B. 1993. The blood-brain barrier: morphology, molecules, and neurothelin. Bioessays. 15:341-346.

34. Morin, O., Patry, P., and Lafleur, L. 1984. Heterogeneity of endothelial cells of adult rat liver as resolved by sedimentation velocity and flow cytometry. J. Cell. Physiol. 119:327-334.

35. DeFouw, D.O. 1988. Structural heterogeneity within the pulmonary microcirculation of the normal rat. Anat. Rec. 221:645-654.

36. Fleming, S., and Jones, D.B. 1989. Antigenic heterogeneity of renal endothelium. J. Pathol. 158:319-323.

37. Rafii, S., et al. 1995. Human bone marrow microvascular endothelial cells support long-term proliferation and differentiation of myeloid and megakaryocytic progenitors. Blood. 86:3353-3363.

38. Stewart, P.A., and Wiley, M.J. 1981. Developing nervous tissue induces formation of blood-brain barrier characteristics in invading endothelial cells: a study using quail-chick transplantation chimeras. Dev. Biol. 84:183-192.

39. Beck, D.W., Vinters, H.V., Hart, M.N., and Cancilla, P.A. 1984. Glial cells influence polarity of the blood-brain barrier. J. Neuropathol. Exp. Neurol. 43:219-224

40. Maxwell, K., Berliner, J.A., and Cancilla, P.A. 1987. Induction of gamma-glutamyl transpeptidase in cultured cerebral endothelial cells by a product released by astrocytes. Brain Res. 410:309-314.

41. Janzer, R.C., and Raff, M.C. 1987. Astrocytes induce blood-brain barrier in endothelial cells. Nature. 325:253-257.

42. Lobrinus, J.A., et al. 1992. Induction of the blood-brain specific HT7 and neurothelin epitopes in endothelial cells of the chick chorioallantoic vessels by a soluble factor derived from astrocytes. Brain Res. Dev. Brain Res. 70:207-211.

43. Zhu, D.Z., Cheng, C.F., and Pauli, B.U. 1991. Mediation of lung metastasis of murine melanomas by a lung-specific endothelial cell adhesion molecule. Proc. Natl. Acad. Sci. USA. 88:9568-9572.

44. Milici, A.J, Furie, M.B., and Carley, W.W. 1985. The formation of fenestrations and channels by capillary endothelium in vitro. Proc. Natl. Acad. Sci. USA. 82:6181-6185.

45. Balligand, J.L., et al. 1995. Nitric oxide-dependent parasympathetic signaling is due to activation of constitutive endothelial (type III) nitric oxide synthase in cardiac myocytes. J. Biol. Chem. 270:14582-14586.

46. Shaul, P.W., et al. 1994. Endothelial nitric oxide synthase is expressed in cultured human bronchiolar epithelium. J. Clin. Invest. 94:2231-2236.

47. Xue, C., Botkin, S.J., and Johns, R.A. 1996. Localization of endothelial NOS at the basal microtubule membrane in ciliated epithelium of rat lung. J. Histochem. Cytochem. 44:463-471.

48. Ogawa, H., et al. 1992. Plasma platelet-derived growth factor levels in coronary circulation in unstable angina pectoris. Am. J. Cardiol. 69:453-456.

49. Ogawa, H., et al. 1993. Platelet-derived growth factor is released into the coronary circulation after coronary spasm. Coron. Artery Dis. 4:437-442.

50. Shaddy, R.E., Hammond, E.H., and Yowell, R.L. 1996. Immunohistochemical analysis of platelet-derived growth factor and basic fibroblast growth factor in cardiac biopsy and autopsy specimens of heart transplant patients. Am. J. Cardiol.77:1210-1215. 